\title{
Adrenal Schwannoma: A Rare Type of Adrenal Incidentaloma
}

${ }^{1}$ Surita Said, ${ }^{2}$ Rohaizak Muhammad, ${ }^{3}$ Hairol A Othman, ${ }^{4}$ Suraya Othman, ${ }^{5}$ Nor FA Rashid, ${ }^{6}$ Reena RM Zin

\begin{abstract}
Benign tumor of the myelin sheath or schwannoma is not uncommon, especially when it involves the peripheral or cranial nerves. But adrenal schwannoma is very rare and commonly presented as an incidentaloma. Adrenal incidentaloma by itself is not uncommon as more imaging is now being performed and its incidence is estimated to be around 4 to $6 \%$. Clinical and imaging studies are not able to differentiate adrenal schwannoma from other causes of incidentaloma, making its diagnosis difficult preoperatively. We report a 64-year-old man who underwent computed tomography (CT) abdomen as part of his health-screening program. An $80 \times$ $70 \mathrm{~mm}$ right adrenal tumor was discovered from the CT with features suggestive of malignancy. He has neither apparent abdominal symptoms nor symptoms related to adrenal hypersecretion. Apart from chronic hypertension, he also suffered from morbid obesity with body mass index (BMI) of 38 . There was no clinical feature to suggest Cushing disease, pheochromocytoma, or primary hyperaldosteronism. His laboratories evaluation including endocrinology studies consistent with a non-functioning adrenal mass. He underwent an uneventful standard transabdominal right adrenalectomy. Immunohistochemistry report showed the tumor has characteristic of Antoni A type and positive for S-100 immunochemistry stain. The diagnosis of adrenal schwannoma was made. This case report highlights the difficulty in the management of adrenal incidentaloma.
\end{abstract}

Keywords: Adrenal, Adrenal carcinoma, Incidentaloma, Nerve sheath, Schwannoma.

How to cite this article: Said S, Muhammad R, Othman HA, Othman S, Rashid NFA, Zin RRM. Adrenal Schwannoma: A Rare Type of Adrenal Incidentaloma. World J Endoc Surg 2017;9(3):111-114.

\section{Source of support: Nil}

Conflict of interest: None

\section{INTRODUCTION}

Adrenal incidentaloma is a mass uncommonly found during imaging studies at autopsy. The disease commonly

\footnotetext{
${ }^{1}$ Medical Officer, ${ }^{2}$ Professor, ${ }^{3-5}$ Surgeon, ${ }^{6}$ Consultant and Senior Lecturer

${ }^{1-5}$ Department of Surgery, Universiti Kebangsaan Malaysia Medical Centre, Kuala Lumpur, Malaysia

${ }^{6}$ Department of Pathology and Diagnostic Laboratory Services Universiti Kebangsaan Malaysia Medical Centre, Kuala Lumpur Malaysia

Corresponding Author: Rohaizak Muhammad, Professor Department of Surgery, Universiti Kebangsaan Malaysia Medical Centre, Kuala Lumpur, Malaysia, Phone: +60391455555, e-mail: Rohaizak@hotmail.com
}

arises either from the adrenal medulla or from the cortex but may arise from any tissue of the adrenal gland. The common benign causes are adrenal hyperplasia and pheochromocytoma. Tumor arising from the nerve sheath of the adrenal gland or adrenal schwannoma is extremely rare. ${ }^{1-3}$ Out of 381,200 autopsy specimen of adrenal incidentalomas examined, only one adrenal schwannoma was detected. ${ }^{4}$ In another study, out of 1,111 cases of adrenal incidentalomas examined, only two cases $(0.18 \%)$ of adrenal schwannoma were detected. ${ }^{5}$ Magnetic resonance imaging and CT imaging are common modalities used to characterize adrenal incidentaloma but difficult due to indistinctive features from other possible diagnosis. Histopathological examination and immunohistochemistry staining are the gold standard in the diagnosis of adrenal schwannoma. ${ }^{1,6}$ Here we present a patient with an adrenal schwannoma that was discovered incidentally as nonfunctioning adrenal incidentaloma.

\section{CASE REPORT}

A 64-year-old Indonesian man with background history of hypertension, diabetes mellitus type II, obesity, and obstructive sleep apnea was referred to our endocrine surgery unit for further evaluation and management of right adrenal mass. He initially went for health screening at another private center. A CT scan of abdomen revealed a huge right adrenal mass with features suggestive of malignancy (Fig. 1). Otherwise, there was no complaint

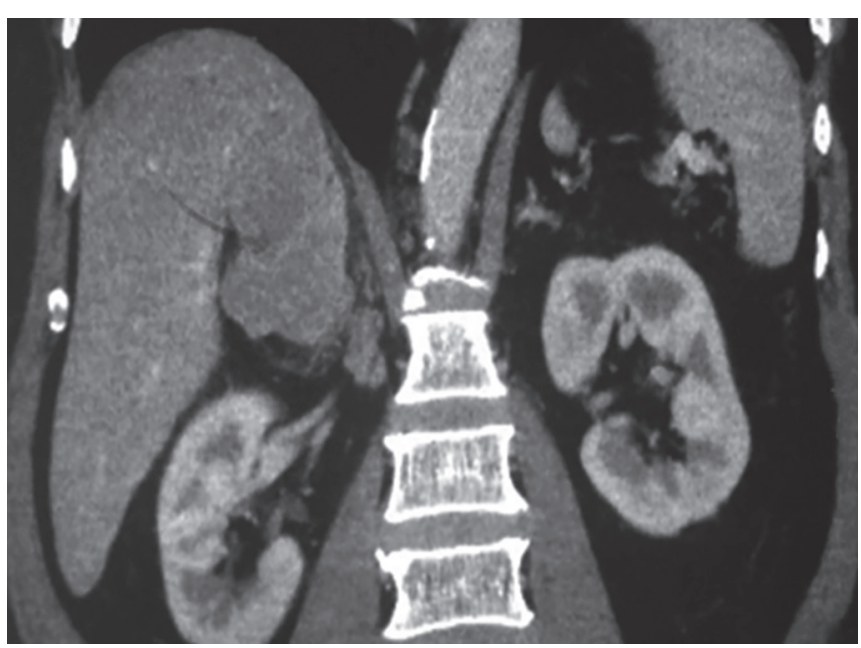

Fig. 1: Sagittal image of CT abdomen shows an irregular heterogeneous tumor suspicious of malignancy arising from the right adrenal gland 


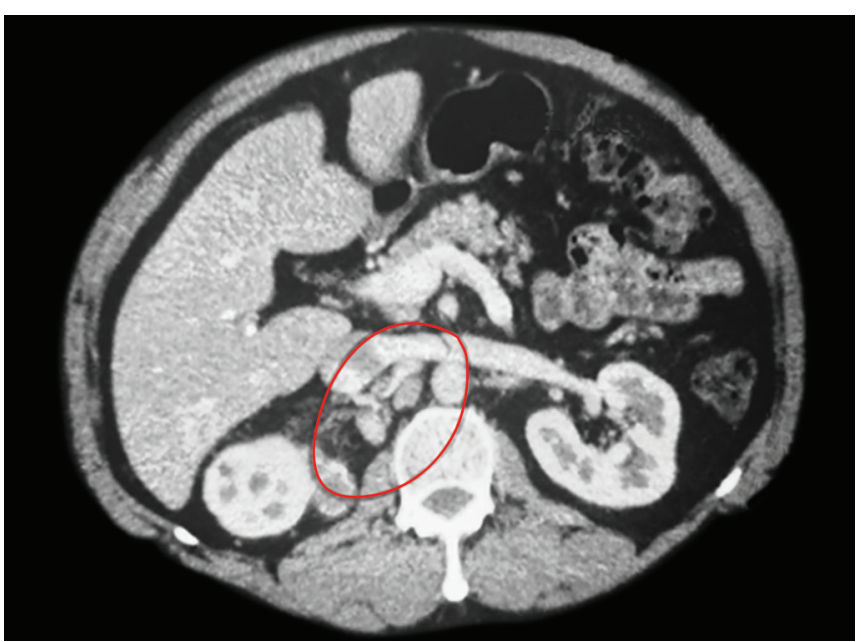

Fig. 2: Multiple enlarged lymph nodes at the aortocaval area

of abdominal pain or discomfort. He also had no constitutional symptoms. He suffered from hypertension since more than 10 years and was currently on two types of antihypertensive medications. His blood pressure was well controlled. During physical examination, patient had no Cushingoid features except for his high BMI of 38. His blood pressure was $140 / 90 \mathrm{~mm} \mathrm{Hg}$. Blood glucose control was in normal range. Abdomen was flabby, no organomegaly, and no mass can be appreciated. Examinations of other systems revealed normal finding.

CT scan abdomen demonstrated an irregular $80 \times 70$ $\mathrm{mm}$ right adrenal mass with a density of $30 \mathrm{HU}$ (Fig. 1) with multiple enlarged aortocaval lymph nodes (Fig. 2). Routine laboratory results were unremarkable with normal potassium level. Endocrinology evaluations of adrenal mass (metanephrine, serum cortisol, dexamethasone suppression test, vanillylmandelic acid) were all within normal range. Due to huge adrenal lesion exhibit features of malignancy from CT images, patient was subjected to an open adrenalectomy. Thus, standard transabdominal right adrenalectomy was performed and complicated with postoperative ileus, which was successfully treated conservatively. He was discharged on postoperative day 5 .

Histology examination showed a $90 \times 70 \times 60 \mathrm{~mm}$ nerve sheath neoplasm, weighing $178.8 \mathrm{gm}$. An area of calcification, hemorrhage, and cystic region was demonstrated. Immunohistochemical analysis showed a uniform spindle cells tumor, which was diffusely positive for S-100 and negative for smooth muscle actin (SMA), desmin, CD34, and epithelial membrane antigen (Fig. 3). A diagnosis of schwannoma was made.
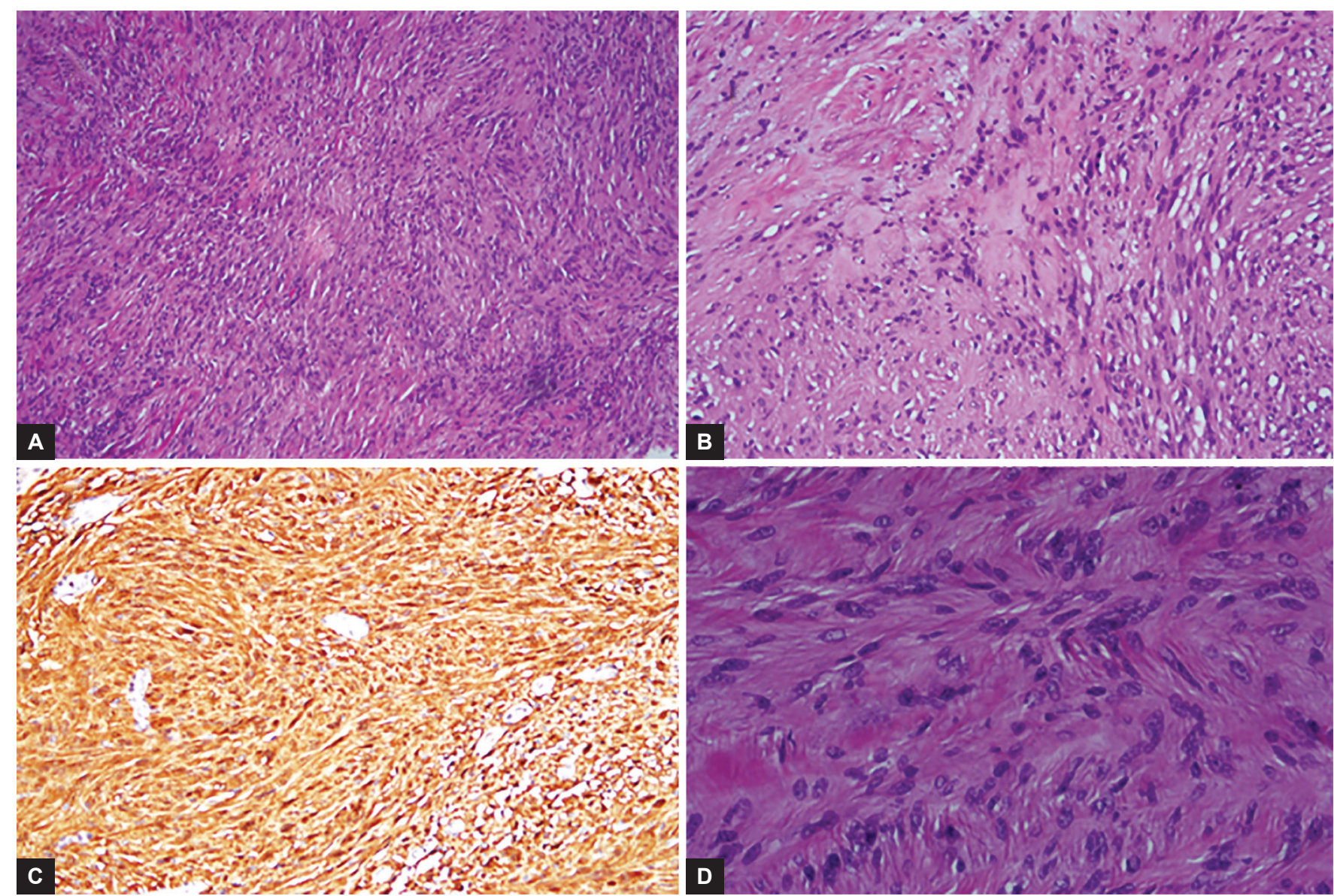

Figs 3A to D: Microscopic view of schwannoma: (A) Hypercellular spindle cells in antoni A area with early formation of verocay body; (B) antoni B areas consisting of spindle cells in loose myxoid matrix; (C) spindle cells positive for S-100 immunohistochemical staining; and (D) hyperchromatin nuclei 


\section{DISCUSSION}

Schwannoma is a tumor arising from the myelin sheath, which was first described by Verocay in 1908 and was later further sub-classified by Antoni in 1920 into two distinct histological patterns. ${ }^{3,6}$ It is commonly seen to arise from the peripheral nerves and cranial nerves at the head and neck region. It rarely involves the visceral organ in the case presented, where it arises from the adrenal medulla. Adrenal schwannoma comprises 1 to $3 \%$ of all schwannomas. ${ }^{1-3}$ Other areas where they can be found include retroperitoneum and juxta-adrenal area. ${ }^{1,3}$ Adrenal schwannoma is thought to arise from Schwann cells from the phrenic nerve, the vagus nerve, or sympathetic trunk that innervate the adrenal medulla. ${ }^{6}$ The explanation for this is related to the embryological development of the adrenal medulla where it is formed by cell migration from the neural crest. Physiologically, if functions as postganglionic neurons. Over the last few years, the number of adrenal schwannomas reported has been increasing, including a large series of 33 cases reported by Mohiuddin and Gilliland. ${ }^{1}$ This could be related to more vigorous use of imaging modalities, such as MRI, CT scan, and PET scan. There is no gender dominance and can occur at any age group. ${ }^{3,5,7}$ Majority of adrenal schwannoma is solitary and benign. Malignant transformation may be found in association with von Recklinghausen disease or neurofibromatosis.,7

Most patients with adrenal schwannomas are asymptomatic as in the case presented. They are also biochemically normal. The possible nonspecific symptoms will be like other adrenal lesions, which is normally abdominal or flank pain or discomfort. ${ }^{1,5,7}$ In the review by Mohiuddin and Gilliland, 13 out of 30 patients presented with symptoms of flank or abdominal discomfort. Others report finding of adrenal schwannoma during investigation for gross hematuria. ${ }^{8}$ The biochemical tests are normal in most cases including serum electrolytes, urinary and serum catecholamines as in the case presented. Patients with retroperitoneal schwannoma secreting noradrenaline have been reported. ${ }^{2,7}$

Adrenal schwannoma on CT image typically appears as a homogeneous, round, and well-circumscribed lesion. Occasionally, it undergoes degenerative changes, such as calcification, hemorrhage, or cystic formation, giving rise to heterogeneous enhancement. ${ }^{3}$ In the case presented, the CT scan showed a huge mass with heterogeneous enhancement, irregular border with possible infiltration to the liver bed, and inferior vena cava, suspicious of adrenal cortical carcinoma. Another imaging test that might be useful in the diagnosis of adrenal schwannoma is 18 F-fluorodeoxyglucose Positron emission tomography CT. The test is able to differentiate between benign and malignant adrenal lesion with sensitivity of 93 to $100 \%$ and specificity of 80 to $100 \%$., 10

The confirmatory diagnosis of adrenal schwannoma is by histological and immunohistochemistry evaluation. Fine needle aspiration cytology for adrenal mass is controversial unless in suspected metastasis. ${ }^{11}$ Histopathological examination of adrenal schwannoma might show both the cellular and the conventional type. ${ }^{1,5}$ A growth pattern is lack of Verocay bodies with increased mitotic activity. 5,12 This appearance might be confused with diagnosis of leiomyoma, ganglioneuroma, or adrenal pheochromocytoma. A conventional type normally shows the characteristic appearance of spindle cells alternating with compact hypocellular (Antoni A) areas and Verocay bodies. In the case presented, microscopic examinations showed evidence of conventional schwannoma with positive immunohistochemistry for S-100 and negative for SMA, desmin, and CD34 which are typical for schwannoma. 1,5,12

Surgical management of adrenal schwannoma follows the adrenal incidentaloma as most of them are asymptomatic and found incidentally. The important features include tumor size, imaging characteristics, and growth rate. Simple rule is to excise all lesions bigger than $6 \mathrm{~cm}$ with close observation for 4 to $6 \mathrm{~cm}$. Any lesion that grows more than $1 \mathrm{~cm}$ over 6 to 12 months is also recommended to be removed. ${ }^{13}$ Our patient's adrenal mass measured $8 \mathrm{~cm}$ with suspicious appearances on CT imaging and warranted surgical excision.

Laparoscopic adrenalectomy is the gold standard for small, benign lesions in the adrenal. The role in the large lesion or suspicious of malignancy is controversial, especially the risk of incomplete resection, local recurrence, and port-site metastases. Large tumor size alone should not be the limiting factor but the presence of local invasion and poor tissue plane on imaging should be taken into consideration for laparoscopic adrenalectomy. ${ }^{14,15}$ In the case presented, an open adrenalectomy was considered in view of large lesion and suspicious features on CT imaging.

\section{CONCLUSION}

Preoperative diagnosis of adrenal schwannoma is difficult due to lack of definitive features on imaging. Definitive diagnosis only established postoperatively by histological and immunohistochemical staining. As majority of them presented as an incidentaloma, the management follows the adrenal incidentaloma.

\section{REFERENCES}

1. Mohiuddin Y, Gilliland MG. Adrenal schwannoma: a rare type of adrenal incidentaloma. Arch Pathol Lab Med 2013 Jul;137(7):1009-1014.

2. Mozafar M, Lotfollahzadeh S, Atqiaee K, Adhamy F. Rare presentation of retroperitoneal schwannoma: a case report. Iran J Cancer Prev 2014 Spring;7(2):107-110. 
3. Adas M, Ozulker F, Adas G, Koc B, Ozulker T, Sahin IM. A rare adrenal incidentaloma: adrenal schwannoma. Case Rep Gastroenterol 2013 Oct;7(3):420-427.

4. Kanthan R, Senger JL, Kanthan S. Three uncommon adrenal incidentalomas: a 13-year surgical pathology review. World J Surg Oncol 2012 Apr;10:64.

5. Kasperlik-Zaluska AA, Roslonowska E, SlowinskaSrzednicka J, Otto M, Cichocki A, Cwikla J, Slapa R, Eisenhofer G. 1,111 patients with adrenal incidentalomas observed at a single endocrinological center: incidence of chromaffin tumors. Ann N Y Acad Sci 2006 Aug;1073:38-46.

6. Jeshtadi A, Govada N, Somalwar SB, Nagulapally S. Schwannoma of adrenal gland. J Med Allied Sci 2014 Jul;4(2):77-80.

7. Ikemoto I, Yumoto T, Yoshino Y, Furuta N, Kiyota H, Oishi Y. Schwannoma with purely cystic form originating from the adrenal area: a case report. Hinyokika Kiyo 2002 May;48(5):289-291.

8. Korets R, Berkenblit R, Ghavamian R. Incidentally discovered adrenal schwannoma. JSLS 2007 Jan-Mar;11(1):113-115.

9. Yun M, Kim W, Alnafisi N, Lacorte L, Jang S, Alavi A. 18FFDG PET in characterizing adrenal lesions detected on CT or MRI. J Nucl Med 2001 Dec;42(12):1795-1799.
10. Tenenbaum F, Groussin L, Foehrenbach H, Tissier F, Gouya H, Bertherat J, Dousset B, Legmann P, Richard B, Bertagna X. 18F-fluorodeoxyglucose positron emission tomography as a diagnostic tool for malignancy of adrenocortical tumours? Preliminary results in 13 consecutive patients. Eur J Endocrinol 2004 Jun;150:789-792.

11. Quayle FJ, Spitler JA, Pierce RA, Lairmore TC, Moley JF, Brunt LM. Needle biopsy of incidentally discovered adrenal masses is rarely informative and potentially hazardous. Surgery 2007 Oct;142(4):497-504.

12. Wick, MR.; Hornick, JL. Immunohistology of soft tissue and osseous neoplasms. In: Dabbs DJ, editor. Diagnostic immunohistochemistry. Philadelphia (PA): Elsevier; 2010. p. 98, 484.

13. NIH state-of-the-science statement on management of the clinically inapparent adrenal mass ("incidentaloma"). NIH Consensus State Sci Statements 2002 Feb;19(2):1-25.

14. Richter KK, Premkumar R, Yoon HS, Mercer P. Laparoscopic adrenalectomy for a rare 14-cm adrenal schwannoma. Surg Laparosc Endosc Percutan Tech 2011 Dec;21(6):e339-e343.

15. Hobart MG, Gill IS, Schweizer D, Sung GT, Bravo EL. Laparoscopic adrenalectomy for large-volume $(>5 \mathrm{~cm})$ adrenal masses. J Endourol 2000 Mar;14(2):149-154. 